\title{
Consumption of oral anticancer drugs in Norway compared by different units of measurements - introduction of new DDDs
}

\author{
Kristine Olsen, Live Storehagen Dansie, Irene Litleskare and Hege Salvesen Blix \\ Department of Drug Statistics, Norwegian Institute of Public Health, Oslo, Norway \\ E-mail: kristine.olsen@fhi.no
}

\begin{abstract}
Background: Antineoplastic agents (ATC group L01) have not been assigned DDDs due to highly interindividual variation in dosages. Consumption data has therefore been presented in other measurement units such as grams of active ingredient. However, the protein kinase inhibitors (PKIs) are a rapidly growing drug group that was introduced to the market recently and are administered orally in a fixed dose. DDDs were therefore established for the PKIs in 2020. In this study we aim to assess whether the newly assigned DDDs would better express drug utilisation patterns in Norway than the current units of measurement.

Methods: Sales data for PKIs (ATC level L01E) by grams, cost, units and packages for 2019 were collected from the Norwegian Drug Wholesales Statistics and data on number of prescriptions and prevalence for 2019 were collected from the Norwegian Prescription Database (NorPD). DDDs were calculated by applying the values of the new DDDs.

Results: The proportions of the different substances varied according to the unit of measurement. DDDs and packages had the highest similarity and correlated better than grams with the prevalence of use in the Norwegian population. BCR-ABL tyrosine kinase inhibitors was the largest group accounting for $31 \%$ of the total consumption (DDD/1000 inhabitants/day) and imatinib was the most sold PKI in all units of measurement except cost.
\end{abstract}

Conclusions: Using an international agreed unit of measurement gives reliability to the study result. Assignment of DDDs to PKIs will improve the quality of drug utilisation studies in this area.

This is an open access article distributed under the Creative Commons Attribution Licence, which permits unrestricted use, distribution, and reproduction in any medium, provided the original work is properly cited.

\section{INTRODUCTION}

The development of new anticancer drugs has steadily increased the latest years and particularly, new targeted orally available substances such as protein kinase inhibitors (PKIs) have enabled an increased number of patients to be treated at home. In 2020, oral substances represented $48 \%$ of the total number of available anticancer drugs (ATC codes) sold in Norway (1).

Drug Utilization Research Group (DURG) was established in 1969 and entrusted with the development of internationally applicable methods for DUR (2), of which one, the Anatomical Therapeutic Chemical (ATC)/Defined Daily Dose (DDD) methodology, has been a cornerstone (3). Products used in the treatment of malignant neoplastic diseases are classified in ATC group L01 Antineoplastic agents (4), but until now no DDDs have been established in this ATC group because of highly individualized use and wide dosage ranges. The recommended dosages also vary substantially based on the type and severity of neoplastic disease.

In Norway, the use of anticancer drugs has been measured in grams. This is a recommended method to be used internationally for these agents (3). A disadvantage by using this method is that drugs with low potency in grams will contribute more to the total amount than drugs with higher potency. Alternative methods could be counting of prescriptions or units (e.g. packages, ampoules or tablets).
In 2018, the WHO International Working Group for Drug Statistics Methodology (ATC/DDD expert group) discussed the possibility of establishing DDDs for oral formulations of substances in L01 Antineoplastic agents as it would be an advantage to follow usage more closely due to the increasing use outside hospitals. It was decided to start with the PKIs since these substances are mainly oral formulations with fixed dosing prescribed to outpatients and often used chronically.

The first PKI classified in L01 was imatinib, which entered the Norwegian market in 2001. Since then many new substances have been introduced. In 2019 there were 58 substances classified as PKIs. The sales of PKIs (measured in grams) in Norway had increased about 35 times since 2001. Because the group had grown extensively, and further growth was expected in the future, the ATC/DDD expert group decided to assign a new ATC 3rd level L01E Protein kinase inhibitors with 13 new 4th levels, subdivided according to the main target for the substances. The proposal for re-classification was through an external consultation process, and the new classification was finally confirmed by the ATC/ DDD expert group in March 2020 and included in the ATC Index 2021 (4). Along with the new subdivision, DDDs for the PKIs were suggested and decided by the ATC/DDD expert group in October 2020 (5). Some of the substances were approved for more than one indication. For these substances the dosage recommendations for the main indication are used as a basis for DDD 
following the WHO definition for DDD: DDD is the assumed average maintenance dose per day for a drug used for its main indication in adults (3). The new DDDs will be included in the ATC/DDD Index in 2022.

The aim of this study is to describe how different units of measurement reflect the therapy pattern of oral protein kinase inhibitors (PKIs), ATC group L01E, in Norway and to evaluate how the newly assigned DDDs correlate with the occurrence of use in the population compared to the currently used units of measurement.

\section{MATERIALS AND METHODS}

Data on sales of medicines in Norway are available in the two national databases; the Norwegian Drug Wholesales Statistics database (1), that provides information on all sales of medicines in Norway, and the Norwegian Prescription Database (NorPD) (6), that includes sales of prescription medicines in primary care, i.e. to all persons living outside institutions in Norway.

PKIs available in Norway, classified in the new ATC $4^{\text {th }}$ levels for PKIs in L01E, were included in the study. In both databases, the following information on oral anticancer drugs included in ATC group L01E Protein kinase inhibitors was collected; generic name and ATC code. Furthermore, from the Norwegian Drug Wholesales Statistics product name and total sales data for 2019 measured in number of tablets/capsules, packs and grams of active ingredient were collected. From the Norwegian Prescription Database the number of prescriptions and prevalence (proportion of users per 1000 inhabitants) in 2019 was gathered.

Use was measured in number of grams, units (tablets or capsules), packages, costs (pharmacy purchase price (AIP) in NOK) and defined daily doses (DDD) per 1000 inhabitants/day and lastly in number of prescriptions/ 1000 inhabitants/year. We calculated DDDs by applying the values of the newly proposed DDDs for oral anticancer drugs available in Norway.

The pattern of use for the different groups of PKIs for cancer was compared by the different units of measurements. The correlation factors between the prevalence and the measurement units DDDs/1000 inhabitants/day, grams/1000 inhabitants/day, packages/1000 inhabitants/day, units/1000 inhabitants/day, cost/1000 inhabitants/day and prescriptions/1000 inhabitants/year for the substances classified in L01E were calculated. The ATC/DDD methodology, version 2021, was used in the analyses.

\section{RESUltS}

Figure 1 shows the therapy patterns in proportions of total consumption of PKIs for anticancer treatment per ATC $4^{\text {th }}$ level by the different units of measurement. Measured in DDD/1000 inhabitants/day, the ATC $4^{\text {th }}$ level L01EA BCR-ABL inhibitors was the largest group accounting for $31 \%$ of the total consumption. L01EX Other protein kinase inhibitors accounted for 19\%,
L01EF CDK-inhibitors 16\%, L01EJ JAK-inhibitors 9\% and L01EL BTK-inhibitors 7\%.

Three groups accounted for considerably less of the total consumption when measured in grams/1000 inhabitants/day compared to DDDs/1000 inhabitants/day. L01EE MEK-inhibitors accounted for $0.1 \%$ in grams/ 1000 inhabitants/day and $2.8 \%$ in DDDs/1000 inhabitants/day, L01EG mTOR-kinase inhibitors accounted for $0.1 \%$ in grams $/ 1000$ inhabitants/day and $4.4 \%$ in DDDs/1000 inhabitants/day and L01EJ JAK-inhibitors accounted for $0.9 \%$ in grams $/ 1000$ inhabitants/day and $8.8 \%$ in DDDs/1000 inhabitants/day. ALK-inhibitors accounted for $7.8 \%$ of the total consumption when measured in grams/1000 inhabitants/day which is considerably more compared to DDDs/1000 inhabitants/day where they accounted for $2.5 \%$.

Table 1 shows therapy patterns in proportions of total consumption of PKIs per ATC $5^{\text {th }}$ level (substance level), classified in ATC group L01E, in Norway in 2019 by different units of measurement. Altogether 39 oral PKI substances were identified. Substances with sale less than $1 \%$ measured in DDDs were excluded from the table (altogether 21 substances, total amount of sale $6.3 \%$ ). When measured in DDDs/1000 inhabitants/day, the five most used PKIs were imatinib, palbociclib, ruxolitinib, ibrutinib and nintedanib, accounting for $54.7 \%$ of the total consumption. Measured in grams/ 1000 inhabitants/day, the five most used PKIs were imatinib, ibrutinib, pazopanib, ribociclib and nintedanib, accounting for $64.9 \%$ of the total consumption.

The proportions of the different substances varied according to unit of measurement. Palbociclib and ruxolitinib had a higher consumption measured in DDDs compared to grams, while pazopanib and ribociclib had a higher consumption measured in grams. Imatinib was the most sold PKI in L01E in all units of measurement except cost.

The correlation with prevalence was best for prescriptions/1000 inhabitants/year $(\mathrm{R}=0,9597)$ then packages/ 1000 inhabitants/day $(\mathrm{R}=0,9195)$, DDDs/1000 inhabitants/day $(\mathrm{R}=0,902)$, units/1000 inhabitants/day $(\mathrm{R}=$ $0,6962)$, cost/1000 inhabitants/day $(R=0,6299)$ and grams/1000 inhabitants/day $(\mathrm{R}=0,5593)$.

\section{DISCUSSION}

Drug utilisation data is always a rough estimate of consumption and never an exact picture of the actual drug use. Further, the reliability of the data depends on the unit of measurement that is being used. DDDs are independent of package size, price, currency and strength and therefore allows for comparisons between population groups and countries (7). Since the DDDs are designed for comparing drug consumption and to follow the trends of drug use over time they do not necessarily reflect the therapeutically equivalent doses, nor dosages given in spesific populations, e.g. pediatric and elderly populations. It is therefore in most cases not appropriate to use the DDDs for issues like reimbursment and 


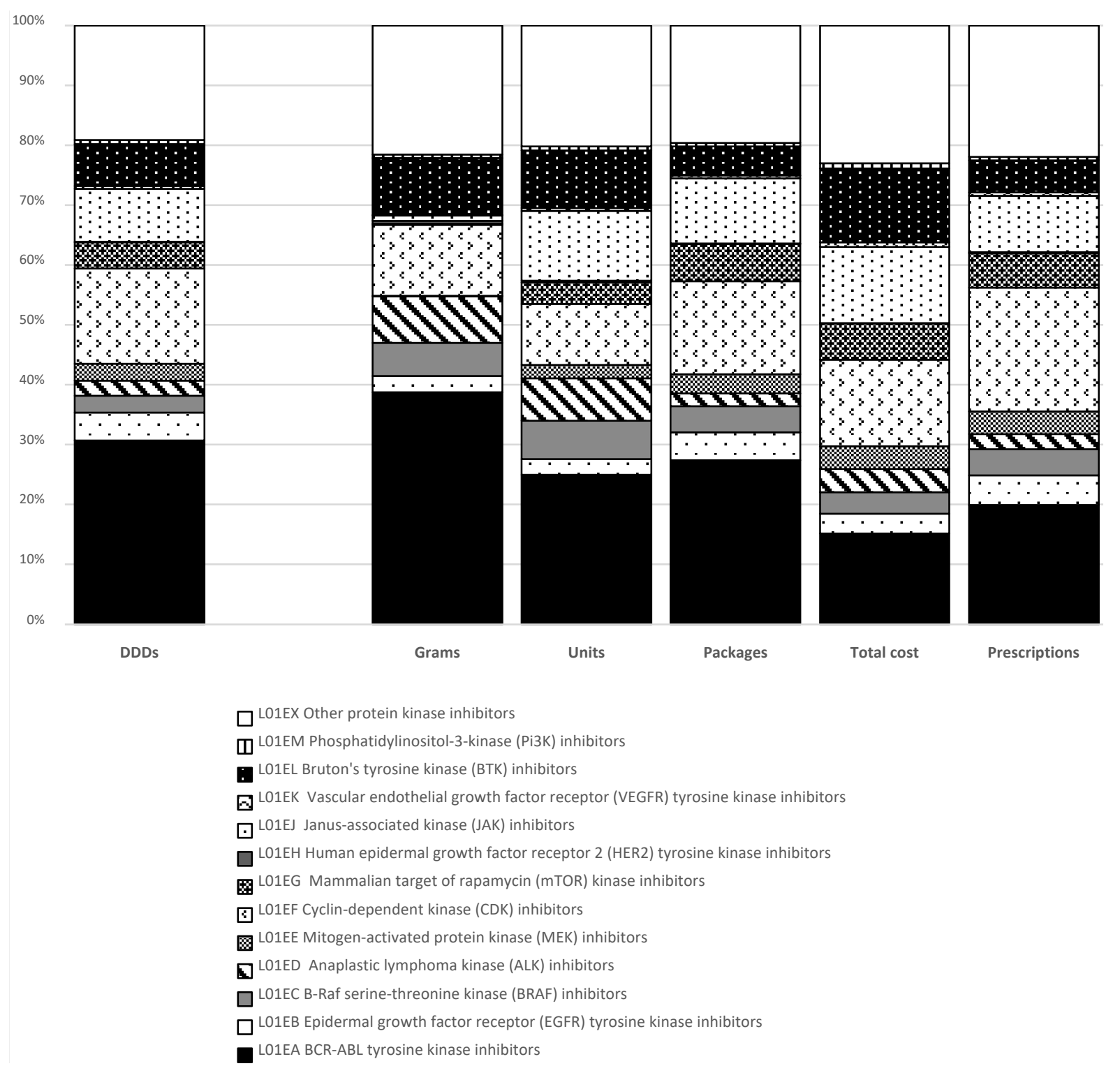

Figure 1. Therapy patterns in proportions of total consumption of oral protein kinase inhibitors (ATC group L01E) by different units of measurement in Norway, 2019.

From the Norwegian Drug Wholesales Statistics (1): Number of defined daily doses (DDDs), Grams, Units (tablets or capsules), Packages (regardless of packet sizes), and Costs (pharmacy purchase prize in NOK) per 1000 inhabitants/day.

From the Norwegian Prescription Database (6): Number of prescriptions dispensed in pharmacy to individuals (regardless of package size and the number of packages dispensed).

pricing e.g. comparing the cost of different drugs or different drug groups (3).

Sometimes alterations of ATC codes and DDDs occur, e.g if the main indication changes or if there are large dose recommendation changes, this implies that drug statistics trends change and historical statistics may be challenging to interpret. It is therefore important to be aware of which version of the ATC index is used in drug consumption studies (3).

Among the different units of measurement applied in this study, DDDs/1000 inhabitants/day corresponded better with number of prescriptions/1000 inhabitants/ year and number of packages/1000 inhabitants/day than grams/1000 inhabitants/day when assessing the therapy patterns in proportions of total consumption for the
ATC $4^{\text {th }}$ level groups of PKIs. This indicates that the new DDDs describe the therapy pattern for the different groups of PKIs more accurately than grams.

The BCR-ABL inhibitors, due to the high consumption of imatinib, was the group of PKIs most used in Norway. This is in line with studies from other countries $(8,9)$. Imatinib is a first line treatment for patients with chronic myeloid leukemia and gastrointestinal stromal tumor (GIST) in Norway $(10,11)$. However, imatinib accounts for only $6 \%$ of the total consumption when using cost as the measurement unit compared to $22.4 \%$ in DDDs. So, although it would give necessary additional information in economic studies, it highlights some of the disadvantages with using an economic unit of measurement in drug consumption studies. Imatinib 
Table 1. Amount of sales in percent of oral protein kinase inhibitors (PKIs), ATC group L01E, in Norway in 2019 by different units of measurement. Indications are the basis for DDDs and are the main indications world-wide. Substances with sale less than $1 \%$ measured in DDDs are excluded.

\begin{tabular}{|c|c|c|c|c|c|c|c|c|c|}
\hline \multirow[b]{2}{*}{ ATC code } & \multirow[b]{2}{*}{ Substance } & \multirow[b]{2}{*}{ ATC 4th level } & \multirow[b]{2}{*}{ Indications } & \multicolumn{6}{|c|}{ Units of measurement } \\
\hline & & & & DDDs $^{1}$ & Grams $^{1}$ & Packages $^{1}$ & Units $^{1}$ & $\operatorname{Cost}^{1}$ & Prescriptions $^{2}$ \\
\hline L01EA01 & imatinib & L01EA BCR-ABL tyrosine kinase inhibitors & CML & 22.4 & 29.3 & 20.1 & 13.9 & 6.0 & 13.7 \\
\hline L01EF01 & palbociclib & L01EF CDK inhibitors & Breast cancer & 9.9 & 3.0 & 9.7 & 3.9 & 9.5 & 13.2 \\
\hline L01EJ01 & ruxolitinib & L01EJ JAK inhibitors & Myelofibrosis & 8.8 & 0.9 & 10.8 & 11.7 & 12.7 & 9.5 \\
\hline L01EL01 & ibrutinib & L01EL BTK inhibitors & CLL & 7.0 & 9.6 & 4.9 & 9.6 & 12.4 & 5.4 \\
\hline L01EX09 & nintedanib & L01EX Other protein kinase inhibitors & NSCLC & 6.6 & 8.2 & 7.2 & 8.4 & 7.3 & 6.0 \\
\hline L01EF02 & ribociclib & L01EF CDK inhibitors & Breast cancer & 6.0 & 8.8 & 5.8 & 6.3 & 4.9 & 7.4 \\
\hline L01EG02 & everolimus & L01EG mTOR kinase inhibitors & $\begin{array}{l}\text { Breast cancer, } \\
\text { RCC, NETs }\end{array}$ & 4.4 & 0.1 & 6.2 & 3.6 & 6.1 & 5.7 \\
\hline L01EX01 & sunitinib & L01EX Other protein kinase inhibitors & GIST, MRCC & 4.1 & 0.4 & 3.9 & 2.2 & 3.8 & 4.9 \\
\hline L01EA03 & nilotinib & L01EA BCR-ABL tyrosine kinase inhibitors & CML & 3.9 & 7.7 & 3.2 & 7.0 & 3.4 & 2.7 \\
\hline L01EX03 & pazopanib & L01EX Other protein kinase inhibitors & RCC, STS & 3.4 & 9.0 & 2.9 & 3.7 & 3.5 & 3.8 \\
\hline L01EA02 & dasatinib & L01EA BCR-ABL tyrosine kinase inhibitors & CML & 3.4 & 1.1 & 2.8 & 3.3 & 3.9 & 2.5 \\
\hline L01EX07 & cabozantinib & L01EX Other protein kinase inhibitors & $\mathrm{RCC}, \mathrm{HCC}$ & 2.7 & 0.5 & 3.2 & 2.1 & 5.6 & 4.2 \\
\hline L01EB01 & gefitinib & L01EB EGFR tyrosine kinase inhibitors & NSCLC & 2.5 & 2.0 & 2.0 & 1.1 & 1.2 & 2.2 \\
\hline L01EE01 & trametinib & L01EE MEK inhibitors & Melanoma & 2.3 & 0.0 & 2.8 & 1.6 & 3.2 & 3.2 \\
\hline L01EC02 & dabrafenib & L01EC BRAF inhibitors & Melanoma & 2.2 & 2.2 & 2.5 & 4.4 & 2.9 & 3.1 \\
\hline L01ED03 & alectinib & L01ED ALK inhibitors & NSCLC & 1.7 & 6.5 & 1.4 & 6.2 & 2.6 & 1.8 \\
\hline L01EX02 & sorafenib & L01EX Other protein kinase inhibitors & $\begin{array}{l}\text { HCC, RCC, Thy- } \\
\text { roid carcinoma }\end{array}$ & 1.2 & 3.1 & 1.0 & 2.2 & 1.4 & 1.3 \\
\hline L01EB02 & erlotinib & L01EB EGFR tyrosine kinase inhibitors & NSCLC & 1.1 & 0.6 & 1.5 & 0.9 & 0.8 & 1.4 \\
\hline
\end{tabular}

Abbreviations: DDD = Defined Daily Dose, $\mathrm{CML}=$ Chronic myeloid leukemia, CDK = Cyclin-dependent kinase, JAK $=$ Janus-associated kinase, BTK $=$ Bruton's tyrosine kinase, CLL $=$ chronic lymphocytic leukaemia, NSCLC $=$ non-small cell lung cancer, mTOR $=$ Mammalian target of rapamycin, $\mathrm{RCC}=$ renal cell carcinoma, NETs $=$ neuroendocrine tumors, GIST $=$ gastrointestinal stromal tumour, MRCC $=$ metastatic renal cell carcinoma, $\mathrm{STS}=$ soft-tissue sarcoma, HCC = hepatocellular carcinoma, EGFR = Epidermal growth factor receptor, $\mathrm{MEK}=\mathrm{Mitogen}-\mathrm{activated}$ protein kinase, $\mathrm{BRAF}=$ B-Raf serine-threonine kinase, ALK = Anaplastic lymphoma kinase, AIP = Pharmacy purchase price/price from wholesaler, NOK = Norwegian kroner

${ }^{1}$ Number of DDDs, Grams, Units (tablets or capsules), Packages (regardless of packet sizes), and Costs (AIP in NOK) per 1000 inhabitants/day are captured from the Norwegian Drug Wholesales Statistics

${ }^{2}$ Number of prescriptions/1000 inhabitants/year dispensed in pharmacy to individuals (regardless of package size and the number of packages dispensed) are captured from the Norwegian Prescription Database (NorPD)

was the only PKI in L01E that had generic formulations available for sale in Norway in 2019 (the indication GIST was still patented). Therefore, imatinib will contribute less to the total consumption pattern in total cost compared to using a weight indicator such as DDDs since generic formulations are much cheaper than original brands. In addition, tender agreements between the health trusts and the pharmaceutical companies on different anticancer treatments may also influence the cost from year to year.

Ruxolitinib, a JAK-inhibitor, was one of the five most sold substances according to all units of measurement assessed in this study, except from grams where it only accounted for $0.9 \%$ of the total sale in Norway. The tablet strengths of ruxolitinib varies from $5 \mathrm{mg}$ to $20 \mathrm{mg}$. Compared with drugs used in much higher doses the amount of ruxolitinib sold is negligible when measured in grams. This example shows that studying drug consumption using grams as the measurement unit may give misleading information because it does not reflect the treatment incidence. Moreover, it can give a false impression of trends in drug consumption for a drug group if a substance requiring much higher doses is substituted by a more potent substance. These potential pitfalls are avoided when using DDD as the measurement unit. Further, the new DDDs had a much higher correlation with prevalence than grams, which indicates that DDDs will describe the consumption of oral anticancer drugs more precisely.
Pharmaceutical companies tend to accommodate their packages according to recommended treatment courses which may explain why the proportions of total consumption measured in DDDs and packages show a similar therapy pattern and that the number of packages sold correlated well with the prevalence of use in the population. However, using packages as an indicator alone to evaluate drug consumption may give incorrect figures if changes in the package size are made during the study period. For example, an increase in the number of units per package could give the false impression that the drug consumption is decreasing. In addition, there could be more than one package size available at the market in a country. There could also be differences in package size among countries, which would make packages unsuitable as a measuring unit when comparing the consumption between countries. This have for example been shown in a comparison study for antibiotic use between France and Belgium (12). Using the number of units to measure the consumption could bring similar challenges, e.g. if there are differents tablet strengths available or if there is a change in the tablet strength during the study period.

Counting of prescriptions could give valuable information in the evaluation of the clinical use of drugs, but is not alone a good unit to measure the drug consumption as we do not know the quantity prescribed (7). In addition, differences in prescribing practice and different reimbursment schemes make this unit of measure- 
ment challenging to use for comparing drug consumption between countries. The new DDDs will enable cross-national comparison and add more information on quantity.

The new classification of the protein kinase inhibitors in L01 made it easier to assign DDDs for most of the substances based on similarities among the substances in one group. However, many of the PKIs have more than one indication and/or more than one dose recommendation for the same indication, such as imatinib, which complicated the assignment of DDDs. The new DDD for imatinib, as well as the other BCR-ABL inhibitors, is based on the treatment of chronic myeloid leukemia when the disease is in its chronic phase corresponding to the standard starting dose. Since the DDD is only a unit of measurement, it does not necessarily reflect the recommended or prescribed daily dose (3). Further, in clinical settings individual patient characteristics and pharmacokinetic considerations are taken into account. In the case for imatinib this may explain why the amount of the total sale was much higher measured in the new DDDs than in number of prescriptions. Generally in drug utilisation studies, when a substance has more than one indication, it should always be assessed whether this would have an impact of the interpretation of data.

However, using an internationally agreed measurement unit gives reliability to the study result. The new DDDs would better describe the consumption pattern of protein kinase inhibitors for cancer treatment in Norway compared to grams which is often used when DDDs are lacking. The newly established DDDs for PKIs will improve the quality of drug utilisation studies.

\section{REFERENCES}

1. Norwegian Drug Wholesales Statistics Database. Norwegian Institute of Public Health, Oslo, Norway.

2. WHO Regional Publications. European Series No. 8. Studies in Drug Utilisation. WHO Europe, Copenhagen, 1979.

3. WHO Collaborating Centre for Drug Statistics Methodology. Guidelines for ATC classification and DDD assignment 2021. Oslo, Norway, 2020. Available from: https://www.whocc.no/atc_ddd_index_and_guidelines/guidelines/(accessed 23.04.2021).

4. WHO Collaborating Centre for Drug Statistic Methodology, ATC classification index with DDD 2021 Oslo, Norway, 2020.

5. WHO Collaborating Centre for Drug Statistics Methodology webpage https://www.whocc.no/ddd/lists_of_temporary_atc_ddds_and_alterations/ddds// (accessed 28 January 2021).

6. Norwegian Prescription Database. Norwegian Institute of Public Health, Oslo, Norway.

7. Elseviers M, Wettermark B, et al. (Eds). Drug Utilization Research Methods and Applications. West Sussex, UK: John Wiley \& Sons, 2016, p. 58-67.

8. Moye-Holz D, Soria Saucedo R, van Dijk JP, Reijneveld SA, Hogerzeil HV. Access to innovative cancer medicines in a middle-income country - the case of Mexico. J Pharm Policy Pract 2018; 11: 25.

9. Leong C, Czaykowski P, Geirnaert M, Katz A, Dragan R, Yogendran M, Raymond C. Outpatient oral anticancer agent utilization and costs in Manitoba from 2003 to 2016: a population-based study. Can J Public Health 2021; 112 (3): 530-540.

10. Norwegian Directorate of Health, Nasjonalt handlingsprogram med retningslinjer for diagnostikk, behandling og oppfølging av sarkom, 2018 Available from: https://www.helsebiblioteket.no/retningslinjer/sarkomer/behandling-av-lokalisert-sykdom/medikamentellbehandling/medikamentell-behandling-av-abdominale-sarkom. (accessed 12.02.2021).

11. Norwegian Directorate of Health, Nasjonalt handlingsprogram med retningslinjer for diagnostikk, behandling og oppfølging av maligne blodsykdommer, 2020 Available from: https://www.helsebiblioteket.no/retningslinjer/maligne-blodsykdommer/kronisk-myelogen-leukemi-kml (accessed 12.02.2021).

12. Watier L, Cavalié P, Coignard B, Brun-Buisson C. Comparing antibiotic consumption between two European countries: are packages an adequate surrogate for prescriptions? Euro Surveill 2017; 22 (46): 17-00352. 